Original Paper http://ajol.info/index.php/ijbcs http://indexmedicus.afro.who.int

\title{
Influence de la composition chimique du sol sur la teneur en éléments nutritifs et le rendement du manioc (Manihot esculenta Crantz, Euphorbiaceae) dans deux zones agro-écologiques du Cameroun
}

\author{
Nono Carine TEMEGNE ${ }^{1,2^{*}}$, Francis NGOME AJEBESONE ${ }^{1}$ et \\ Apollin FOTSO KUATE ${ }^{3}$
}

${ }^{I}$ Institut de la Recherche Agricole pour le Développement (IRAD), BP : 2123 Yaoundé, Cameroun.

${ }^{2}$ Université de Yaoundé I, Faculté des Sciences, Département Biologie et Physiologie Végétales, $B P$ : 812 Yaoundé, Cameroun.

${ }^{3}$ Institut International d'Agriculture Tropicale (IITA), BP: 2008 Yaoundé, Cameroun.

*Auteur correspondant ; Email: nonocarine2003@yahoo.fr ; Tel : (+237)697076 464.

\section{REMERCIEMENTS} Cameroun.

Cette recherche a été financée par le projet C2D PAR Manioc dont le point focal se trouve à l'IRAD du

\section{RÉSUMÉ}

L'étude a été menée dans l'objectif d'évaluer l'influence de la composition chimique du sol sur la teneur en éléments nutritifs et le rendement du manioc dans deux zones agro-écologiques du Cameroun. L'échantillonnage s'est fait à Ekona (zone 4) et Ongot (zone 5). Une variété de manioc améliorée (8034) et deux variétés locales (Local Red et Bassa Noire) âgées de 12 mois ont été utilisées. Les résultats ont montrés que le sol d'Ekona (andosol) est significativement plus riche en éléments minéraux (azote total: 1,34\% ; phosphore disponible: $15 \mathrm{mg} \cdot \mathrm{kg}^{-1}$ ) que celui d'Ongot [(ferralsol), (azote total: 0,11\% ; phosphore disponible: 4 mg.kg $\left.{ }^{-1}\right)$ ]. La variété 8034 produit significativement plus de biomasse fraîche totale et de rendement en tubercules que les variétés locales. Elle est significativement plus riche en éléments minéraux que les variétés locales dans les deux sites. Les éléments minéraux contenus dans les feuilles de manioc sont très fortement corrélés $\left(\mathrm{r}^{2}: 0,999, \mathrm{p}<0,001\right)$ à ceux contenus dans le sol. La richesse des feuilles et des tubercules de manioc en éléments nutritifs est donc proportionnelle à celle du sol en éléments minéraux. Ce résultat préliminaire constitue une avancée dans la détermination des doses de fertilisants à recommander pour la culture du manioc au Cameroun.

(C) 2015 International Formulae Group. All rights reserved.

Mots clés : Zones agro-écologiques, éléments minéraux, analyse des sols, manioc, rendement. 


\title{
Effect of soil chemical composition on nutrient uptake and yield of cassava (Manihot esculenta Crantz, Euphorbiaceae) in two agro-ecological zones of Cameroon
}

\begin{abstract}
A study was conducted to evaluate the effect of soil chemical composition on nutrient uptake and yield of cassava in two agro-ecological zones of Cameroon. Soil and plant samples were obtained from twelve month old pure culture cassava fields at Ekona (zone 4) and Ongot village (zone 5). An improved variety (8034) and two local varieties (Local Red at Ekona and Bassa Noire at Ongot) were used. Results showed that the soil from Ekona (Andosol) was significantly higher in nutrients (total nitrogen: 1.34\%; phosphorus available: $15 \mathrm{mg} \cdot \mathrm{kg}^{-1}$ ) than the soil from Ongot [(Ferralsols), (total nitrogen: 0.11\%; phosphorus available: 4 mg. $\left.\mathrm{kg}^{-1}\right)$ ]. The improved variety accumulated significantly more total fresh biomass and root yield than the local varieties, irrespective of the site. Similarly, nutrient uptake was higher with the improved variety across the zones. A positive and highly significant correlation $\left(\mathrm{r}^{2}: 0.999, \mathrm{p}<0.001\right)$ was observed between nutrient uptake by cassava and soil nutrient availability. Thus, it may be concluded that the quantity of nutrient in the leaves and roots of cassava is proportionate to the quantity of nutrient elements in the soil. This preliminary result constitutes a solid base for the determination of an appropriate fertilizer scheme for cassava cultivation in Cameroon.

(c) 2015 International Formulae Group. All rights reserved.
\end{abstract}

Keywords : Agro-ecological zones, nutrients, soil analyses, cassava, yield.

\section{INTRODUCTION}

Les sols des pays d'Afrique subsaharienne ont un faible niveau de fertilité inhérente (FAO, 2003) associé à des contraintes naturelles spécifiques pour chaque zone agro-écologique. Ces carences sont des facteurs clés de la production. Bien que le manioc (Manihot esculenta) soit connu pour croître et produire dans les sols pauvres, sa culture nécessite une richesse minimale en éléments nutritifs du sol pour un rendement acceptable.

En effet, le manioc est l'aliment de base d'environ 800 millions de personnes à travers le monde (FAO, 2000 ; Lebot, 2009). Il est consommé pour ses feuilles et ses racines (Betiku et al., 2011 ; Gnonlonfin et al., 2011 ; Famurewa et al., 2013 ; Koko et al., 2014). D'après la FAO, le Cameroun a produit 4287177 tonnes de manioc (tubercules) en
2013 (FAO, 2015). Le manioc est cultivé dans quatre des cinq zones agroécologiques du Cameroun sur environ 205 000 ha et est l'une des cultures vivrières les plus importantes. Il constitue $80 \%$ des tubercules dans la zone de la forêt (IRAD, 2013). Chaque ménage camerounais consomme environ $75 \mathrm{~kg} \cdot \mathrm{an}^{-1}$ de produits dérivés des racines tubéreuses de manioc (IRAD, 2013). A Yaoundé, la consommation totale de manioc est de $63 \mathrm{~kg} \cdot$ personne $/ 1 / \mathrm{an}$ (Dury, 2001). Il est la première source d'hydrates de carbone dans toute la moitié sud du pays (Tricoche et al., 2008). Le manioc et ses produits occupent la première place avec $43 \%$ des parts de marché des racines et tubercules, $26 \%$ pour les produits transformés et $17 \%$ pour les racines fraîches (PNDRT, 2003). 
Depuis la fin des années 1980, la production de tubercules de manioc a diminué et les prix des dérivés du manioc ont augmenté dans les marchés urbains (IRAD, 2013). En outre, la pauvreté des sols, la faible diffusion des variétés améliorées à haut rendement résistantes aux maladies et aux ravageurs du manioc figurent parmi les causes de cette baisse de la production de manioc. Les agriculteurs ont besoin de soutien pour diagnostiquer, corriger les carences minérales $\mathrm{du}$ sol et augmenter la production de racines tubéreuses. Les études antérieures réalisées sur le manioc ont montré que les éléments minéraux (l'azote, le phosphore, le potassium, le calcium, le magnésium) pouvaient être des bons indicateurs de la qualité des sols (Ngome et al., 2013). Une voie prometteuse serait donc la compréhension de la relation entre la composition en éléments nutritifs du sol et ceux de la plante. L'objectif du travail était de déterminer l'effet de la composition chimique $\mathrm{du}$ sol sur le rendement et la composition nutritive des feuilles de manioc dans les Régions du Centre (village Ongot, Mbankomo) et du Sud-Ouest (Ekona) du Cameroun.

\section{MATÉRIEL ET MÉTHODES Site d'étude}

La collecte des échantillons de plantes de manioc a été faite dans deux zones agroécologiques du Cameroun au cours de la campagne agricole 2015 (Figure 1).

Le site d'Ekona (N 04 ${ }^{\circ} 12^{\prime} 741 "$, E $009^{\circ} 19^{\prime} 755^{\prime \prime}$, altitude : $452 \mathrm{~m}$ ) appartient à la Région du Sud-Ouest. Il est localisé dans la zone de forêt humide à pluviométrie monomodale. Il est caractérisé par un sol volcanique (andosol) et des précipitations de 3 $076 \mathrm{~mm}$ par an. La température moyenne de l'air varie de 19 à $23^{\circ} \mathrm{C}$. Le climat est de type "camerounien", très humide et chaud, variante du climat équatorial (Tsalefac et al., 2003 ; IRAD, 2008).

Le site d'Ongot (N 03 51' 292", E $011^{\circ} 22^{\prime} 568^{\prime \prime}$, altitude : $794 \mathrm{~m}$ ), de la localité de Mbankomo est situé dans la zone agroécologique de forêt humide à pluviométrie bimodal, à environ $18 \mathrm{~km}$ de Yaoundé (Région du Centre). La température moyenne journalière de l'air varie de 23 à $24{ }^{\circ} \mathrm{C}$ (Atangana, 1998). Il est caractérisé par des précipitations de $1617 \mathrm{~mm}$ par an. Ongot est également caractérisé par des sols ferralitiques (ferralsols), généralement acides. La zone est régie par un climat de type équatorial guinéen avec quatre saisons : une grande saison de pluie de septembre à novembre, une longue saison sèche de décembre en février, une courte saison de pluie de mars en juin et une courte saison sèche de juillet à août (IRAD, 2008 ; Ngome et al., 2013).

Dans chaque site trois champs de manioc ont été échantillonnés. Dans chaque champ de manioc, dix échantillons de sol ont été prélevés de 0 à $15 \mathrm{~cm}$ de profondeur en dessous des pieds de manioc, à l'aide d'une tarière selon la méthode de transect décrite par Okalebo et al. (2002). Les échantillons de sol prélevés ont été ensuite séchés à la température ambiante du laboratoire durant deux semaines environ. Puis, ils ont été tamisés avec un tamis de mailles $2 \mathrm{~mm}$, et ont enfin été conservés à la température du laboratoire $\left(\sim 25{ }^{\circ} \mathrm{C}\right)$ dans des sachets en plastique appropriés jusqu'à l'analyse. Pour chaque site, les analyses du sol ont été faites sur $200 \mathrm{~g}$ d'homogénat de sol au Laboratoire d'Analyse des Plantes, Sols, Engrais et Eaux (LAPSEE) de l'Institut de la Recherche Agricole pour le Développement (IRAD) de Nkolbisson (Cameroun). Le pH a été mesuré avec une électrode en verre en utilisant le ratio sol/eau de 1/2,5. Le carbone organique (CO) a été déterminé par la méthode de combustion humide de Walkley et Black. La matière 
organique (MO) a été calculée par la formule : MO = CO*1,72 (Okalebo et al. (2002). L'azote total a été déterminé par la méthode de Kjehldahl. Le potassium a été dosé par le spectrophotomètre d'absorption atomique. Toutes les analyses effectuées ont été faites suivant les procédures décrites par Okalebo et al. (2002). Le Tableau 1 présente les résultats de l'analyse chimique des sols des deux sites.

\section{Matériel végétal}

Le matériel végétal est constitué de trois variétés de manioc de 12 mois d'âge dont deux variétés locales (Local Red et Bassa Noire) et une variété améliorée (8034). La 8034 est une variété améliorée développée par l'IRAD.

\section{Echantillonnage}

Dans chaque site, dix plants de manioc de chaque variété ont été récoltés, les feuilles et les racines ont été séparées des tiges. Chaque partie de la plante a été pesée pour obtenir sa biomasse fraîche. Les feuilles de manioc ont été ensuite séchées.

\section{Dosage des éléments minéraux}

Après séchage, la teneur des feuilles de manioc en éléments minéraux $(\mathrm{N}, \mathrm{Ca}, \mathrm{Mg}, \mathrm{K}$, $\mathrm{P}, \mathrm{Zn}$ et $\mathrm{Fe}$ ) a été analysée au LAPSEE. Les analyses effectuées ont été faites suivant les procédures décrites par Okalebo et al. (2002). Seuls les éléments minéraux présents dans les feuilles de manioc ont été dosés. En effet, les travaux réalisés par le CIAT (Centre International de l'Agriculture Tropicale) admettent que les proportions à peu près égales de $\mathrm{P}$ (phosphore) sont trouvés dans les racines et les tiges de manioc, tandis qu'environ $60 \%$ de potassium sont trouvés dans les racines de manioc et seulement $40 \%$ dans les tiges et les feuilles de manioc tombées (Howeler et Phien, 2000). La quantité d'azote présente dans la tige feuillée est d'environ $75 \%$ contre $25 \%$ contenu dans les racines tubéreuses du manioc (Howeler et Phien, 2000).

\section{Analyse statistique des données}

Les données obtenues ont été traitées par une analyse de variance (ANOVA) à un critère de classification à l'aide du logiciel MINITAB version 16. Le test de structuration des moyennes de Newman-keuls au seuil 5\% a permis de classer les moyennes et identifier celles qui sont différentes. Le test de corrélation de Pearson a été utilisé pour analyser les corrélations entre la composition en éléments minéraux des sols et celle des feuilles de manioc.

\section{RÉSULTATS \\ Rendements}

La Figure 2 illustre la biomasse fraîche totale ainsi que le rendement en racines tubéreuses du manioc à Ekona et à Ongot. Dans les deux sites, la biomasse fraîche totale $(\mathrm{p}<0,01)$ et le rendement en tubercules $(\mathrm{p}<0,001)$ de manioc sont significativement plus élevés chez la variété améliorée 8034. Ainsi, la biomasse fraîche totale de la 8034 est trois fois plus élevée que celle de la Bassa Noire à Ongot et environ cinq fois plus élevée que celle de la Local Red à Ekona. Le rendement des tubercules de manioc de la 8034 à Ekona $\left(57000 \pm 9500^{\mathrm{a}} \mathrm{kg} \cdot \mathrm{ha}^{-1}\right)$ et à Ongot $\left(35000 \pm 3000^{\mathrm{b}} \quad \mathrm{kg} \cdot \mathrm{ha}^{-1}\right)$ dépasse largement ceux de la Local Red $\left(12800 \pm 1900^{\circ}\right.$ $\left.\mathrm{kg} \cdot \mathrm{ha}^{-1}\right)$ et de la Bassa Noire $\left(10400 \pm 500^{\mathrm{c}}\right.$ $\left.\mathrm{kg} \cdot \mathrm{ha}^{-1}\right)$. Ces deux paramètres du rendement sont significativement $(\mathrm{p}<0,05) \quad$ plus importants à Ekona qu'à Ongot.

\section{Eléments minéraux prélevés par les feuilles}

Comparées à celles d'Ongot (Tableau 2), les feuilles de manioc cultivées à Ekona sont significativement plus riches en azote $(\mathrm{N})$, calcium (Ca), magnésium ( $\mathrm{Mg})$, 
potassium (K), phosphore $(\mathrm{P})$ et $\mathrm{Fe}(\mathrm{Fer})$. Ainsi, les feuilles de la variété 8034 à Ekona $(1,040 \%)$ sont significativement plus riches en $\mathrm{K}$ que celle d'Ongot $\left(0,956^{\mathrm{b}} \%\right)$. Le zinc (Zn) est cependant différemment prélevé par rapport aux autres éléments minéraux. En effet, les feuilles de manioc de la variété 8034 récoltées dans le site d'Ongot $\left(53,102^{\mathrm{a}} \mu \mathrm{g} \cdot \mathrm{g}^{-1}\right)$ sont plus riches en $\mathrm{Zn}$ que celles récoltées à Ekona $\left(49,429^{\mathrm{b}} \mu \mathrm{g} \cdot \mathrm{g}^{-1}\right)$.

Dans les deux sites, les feuilles de la variété améliorée 8034 sont significativement $(\mathrm{p}<0,001)$ plus riches en éléments minéraux $(\mathrm{N}, \mathrm{Ca}, \mathrm{Mg}, \mathrm{K}, \mathrm{P}$, Fer et $\mathrm{Zn}$ ) que celles des variétés locales (Local Red, Bassa Noire). Les feuilles de la variété 8034 produites à Ekona $\left(0,429^{\mathrm{a}} \%\right)$ et à Ongot $\left(0,415^{\mathrm{b}} \%\right)$ sont ainsi significativement plus riches en $\mathrm{Mg}$ que celles des variétés locales (Bassa Noire $\left(0,382^{\mathrm{d}} \%\right)$ et Local Red $\left.\left(0,390^{\mathrm{c}} \%\right)\right)$.

\section{Tests de corrélation}

Les paramètres de rendement (biomasse fraîche totale et rendement des tubercules) sont très fortement $(p<0,01)$ corrélés entre eux (Tableau 3). Le rendement en tubercule de manioc $\left(\mathrm{kg} \cdot \mathrm{ha}^{-1}\right)$ est corrélé $(\mathrm{p}<0,05)$ aux éléments minéraux [azote total $\left(r^{2}: 0,886\right)$, calcium $\left(r^{2}: 0,884\right)$, magnésium $\left(r^{2}: 0,866\right)$, potassium $\left(r^{2}: 0,888\right)$, phosphore $\left(r^{2}: 0,889\right)$, zinc $\left(r^{2}:-0,885\right)$ et fer $\left.\left(r^{2}: 0,886\right)\right]$ contenus dans les feuilles de manioc ainsi qu'aux caractéristiques $\left[\mathrm{pH}-\mathrm{H}_{2} \mathrm{O}\left(\mathrm{r}^{2}: 0,896\right)\right.$, $\mathrm{pH}-\mathrm{HCl}\left(\mathrm{r}^{2}: 0,896\right)$, carbone organique $\left(\mathrm{r}^{2}\right.$ : $0,887)]$ et aux éléments minéraux du sol [azote total $\left(r^{2}: 0,884\right)$, phosphore $\left(r^{2}: 0,884\right)$, potassium $\left(r^{2}: 0,888\right)$, calcium $\left(r^{2}: 0,885\right)$, sodium $\left.\left(r^{2}: 0,877\right)\right]$. A l'exception du rendement en tubercule de manioc, la biomasse fraîche totale n'est corrélée à aucun autre paramètre étudié.

Globalement, les éléments minéraux contenus dans les feuilles (azote total, calcium, magnésium, potassium, phosphore, zinc et fer) sont très fortement corrélés $(p<0,001)$ entre eux (Tableau 3). Ainsi, le contenu en azote total des feuilles est très fortement corrélé aux contenus en calcium $\left(\mathrm{r}^{2}\right.$ : $0,999)$, magnésium $\left(r^{2}: 0,992\right)$, potassium $\left(r^{2}\right.$ : $0,999)$, phosphore disponible $\left(r^{2}: 0,995\right)$, fer $\left(r^{2}: 0,999\right)$ des feuilles et négativement corrélé au contenu en zinc $\left(r^{2}:-0,999\right)$ des feuilles de manioc. Ils sont également fortement corrélés $(\mathrm{p}<0,001)$ à la quantité d'éléments minéraux présents dans le sol (azote total, phosphore, potassium, calcium, sodium), ainsi qu'au $\mathrm{pH}$ et à la teneur en carbone organique du sol des deux sites. La quantité d'azote total contenue dans les feuilles de manioc est très fortement corrélée à l'azote total $\left(\mathrm{r}^{2}: 0,999\right)$, au phosphore disponible $\left(r^{2}: 0,999\right)$, au potassium $\left(r^{2}: 0,999\right)$, au calcium $\left(r^{2}: 0,999\right)$, au sodium $\left(r^{2}: 0,953\right)$ du sol. Elle est également corrélée au $\mathrm{pH}\left(\mathrm{r}^{2}: 0,896\right)$ et à la teneur en carbone organique $\left(r^{2}: 0,887\right) d u$ sol.

Les éléments minéraux contenus dans le sol (azote total, phosphore, potassium, calcium, sodium), l'acidité du sol $\left(\left(\mathrm{pH}-\mathrm{H}_{2} \mathrm{O}\right.\right.$, $\mathrm{pH}-\mathrm{HCl}$ ) et la teneur en carbone organique du sol sont également très fortement corrélés $(p<0,001)$ entre eux (Tableau 3$)$ dans les deux sites d'étude. Ainsi, la quantité d'azote total du sol est très fortement corrélée au phosphore disponible $\left(r^{2}: 0,999\right)$, au potassium $\left(r^{2}\right.$ : $0,999)$, au calcium $\left(r^{2}: 0,999\right)$, au sodium $\left(r^{2}\right.$ : $0,946)$, et à la teneur en carbone organique du sol $\left(r^{2}: 0,999\right)$. 
N. C. TEMEGNE et al. / Int. J. Biol. Chem. Sci. 9(6): 2776-2788, 2015

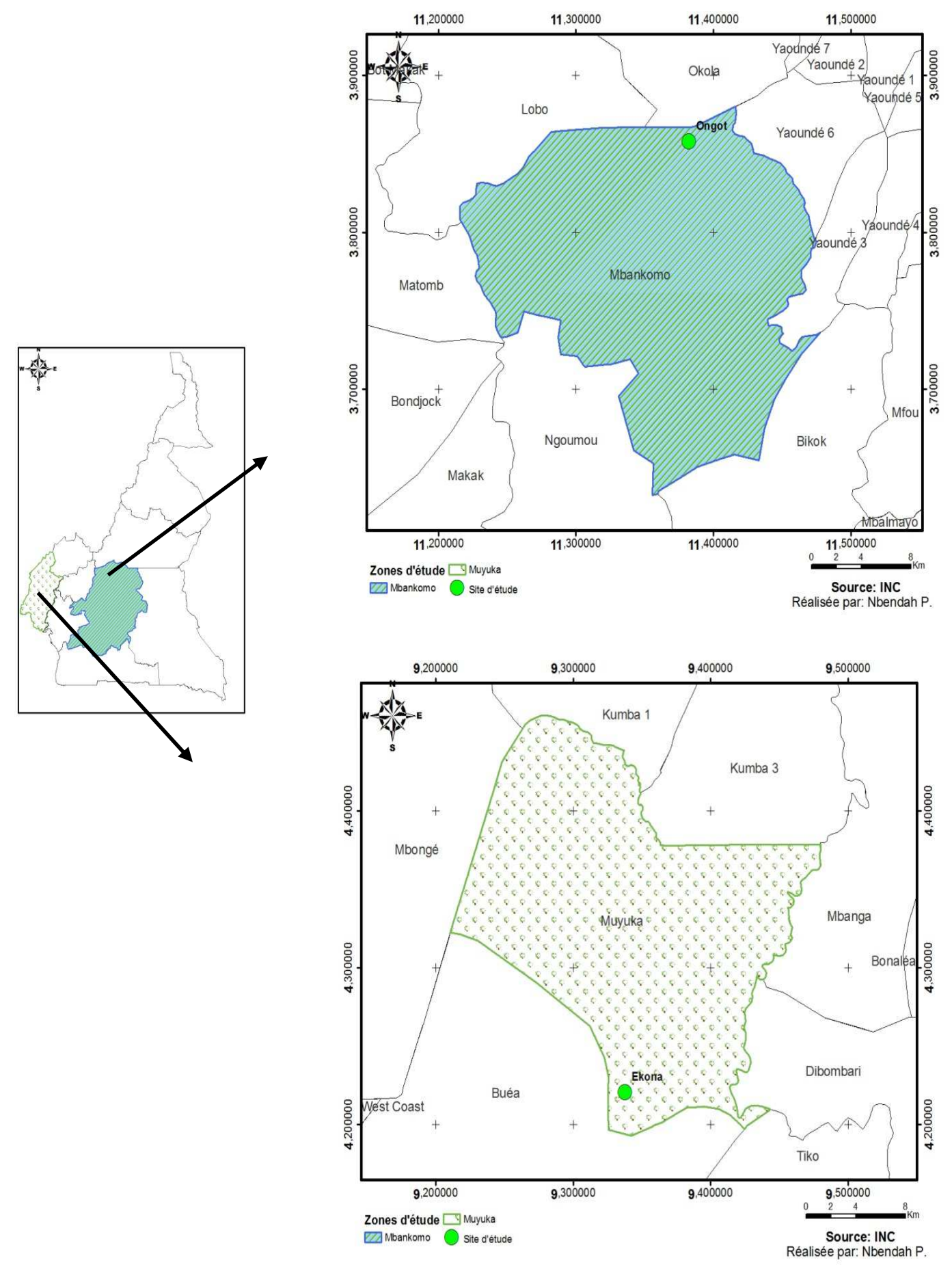

Figure 1: Sites d'étude dans les Régions du Centre (Ongot) et du Sud-Ouest (Ekona). 


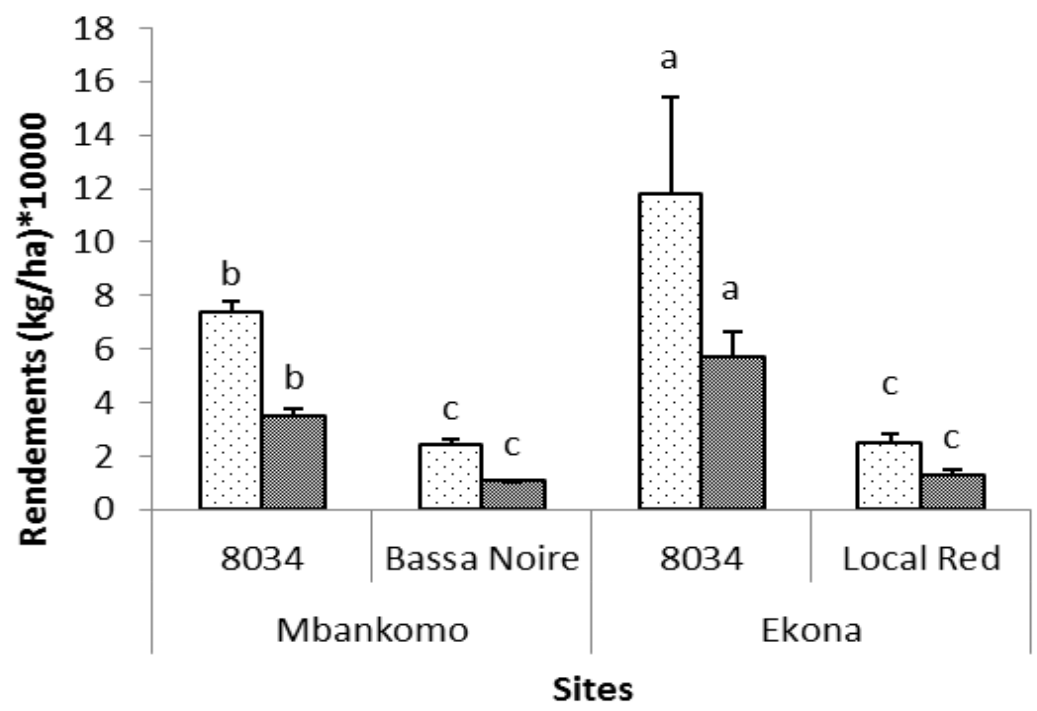

$\square$ Biomasse totale $\square$ Tubercules

Figure 2: Rendements des variétés de manioc récoltées à Ekona et Mbankomo. Les valeurs des histogrammes portant la même lettre ne sont pas significativement différentes au seuil de $5 \%$ du test de NewmanKeuls.

Tableau 1: Analyse chimique des sols dans les zones d'étude (Ekona et Ongot).

\begin{tabular}{|c|c|c|}
\hline \multirow{2}{*}{ Caractéristiques du sol } & \multicolumn{2}{|c|}{ Sites } \\
\hline & Ongot & Ekona \\
\hline $\mathrm{pH}\left(\mathrm{H}_{2} \mathrm{O}\right)$ & 4,7 & 5,47 \\
\hline $\mathrm{pH}(\mathrm{KCl})$ & 3,8 & 4,71 \\
\hline $\mathrm{CO}(\%)$ & 1,82 & 3,33 \\
\hline N Total (\%) & 0,11 & 1,34 \\
\hline $\mathrm{C} / \mathrm{N}$ & 16,55 & 2,49 \\
\hline MO & 3,13 & 5,73 \\
\hline P disponible (mg/kg) & 4 & 15 \\
\hline $\mathrm{K}^{+}(\mathrm{cmol} / \mathrm{kg})$ & 0,15 & 0,86 \\
\hline $\mathrm{Ca}^{2+}(\mathrm{cmol} / \mathrm{kg})$ & 0,4 & 4,3 \\
\hline $\mathrm{Na}^{+}(\mathrm{cmol} / \mathrm{kg})$ & 0,03 & 0,08 \\
\hline $\mathrm{Mg}^{2+}(\mathrm{cmol} / \mathrm{kg})$ & - & 1,61 \\
\hline $\mathrm{Al}^{3+}(\mathrm{cmol} / \mathrm{kg})$ & - & 0,35 \\
\hline CEC à pH : $7(\mathrm{cmol} / \mathrm{kg})$ & - & 7,35 \\
\hline
\end{tabular}

$\mathrm{pH}$ : potentiel d'hydrogène, $\mathrm{KCl}$ : chlorure de potassium, $\mathrm{H}_{2} \mathrm{O}$ : oxyde d'hydrogène, $\mathrm{CO}$ : carbone organique, $\mathrm{N}$ : azote, $\mathrm{C} / \mathrm{N}$ : ratio carbone/azote, $\mathrm{MO}:$ matière organique $(\mathrm{CO} * 1,72), \mathrm{P}:$ phosphore, $\mathrm{K}:$ potassium, $\mathrm{Ca}:$ calcium, $\mathrm{Na}$ : sodium, $\mathrm{Mg}$ : magnésium, Al : aluminium, $\mathrm{CEC}$ : capacité d'échange cationique. Source : IRAD 
N. C. TEMEGNE et al. / Int. J. Biol. Chem. Sci. 9(6): 2776-2788, 2015

Tableau 2: Composition des feuilles de manioc en éléments minéraux à Ekona et à Ongot.

\begin{tabular}{lclllllll}
\hline \multicolumn{2}{c}{ Echantillonnage } & \multicolumn{7}{c}{ nutriments prélevés des feuilles } \\
\hline \multirow{2}{*}{ Sites } & Variétés & N total $(\%)$ & Ca $(\%)$ & Mg $(\%)$ & K $(\%)$ & $\mathbf{P}(\%)$ & $\mathbf{Z n}(\boldsymbol{\mu g} / \mathbf{g})$ & $\mathbf{F e}(\boldsymbol{\mu g} / \mathbf{g})$ \\
\hline \multirow{2}{*}{ Mbankomo } & 8034 & $3,825 \mathrm{~b}$ & $1,633 \mathrm{~b}$ & $0,415 \mathrm{~b}$ & $0,956 \mathrm{~b}$ & $0,271 \mathrm{~b}$ & $53,102 \mathrm{a}$ & $186,500 \mathrm{~b}$ \\
& Bassa Noire & $3,519 \mathrm{~d}$ & $1,502 \mathrm{~d}$ & $0,382 \mathrm{~d}$ & $0,880 \mathrm{~d}$ & $0,249 \mathrm{~d}$ & $48,854 \mathrm{c}$ & $171,580 \mathrm{~d}$ \\
\hline \multirow{2}{*}{ Ekona } & 8034 & $4,021 \mathrm{a}$ & $1,769 \mathrm{a}$ & $0,429 \mathrm{a}$ & $1,040 \mathrm{a}$ & $0,288 \mathrm{a}$ & $49,429 \mathrm{~b}$ & $196,779 \mathrm{a}$ \\
& Local Red & $3,659 \mathrm{c}$ & $1,610 \mathrm{c}$ & $0,390 \mathrm{c}$ & $0,946 \mathrm{c}$ & $0,262 \mathrm{c}$ & $44,980 \mathrm{~d}$ & $179,069 \mathrm{c}$ \\
\hline
\end{tabular}

N: Azote, Ca: Calcium, Mg: Magnésium, K: Potassium, P: phosphore, Zn: Zinc, Fe: Fer. Pour chaque nutriment, les valeurs suivies

de la même lettre ne sont pas significativement différentes au seuil $5 \%$ au test de Newman-keuls.

Tableau 3: Corrélation entre les paramètres étudiés pour la variété améliorée 8034 à Ekona et à Ongot.

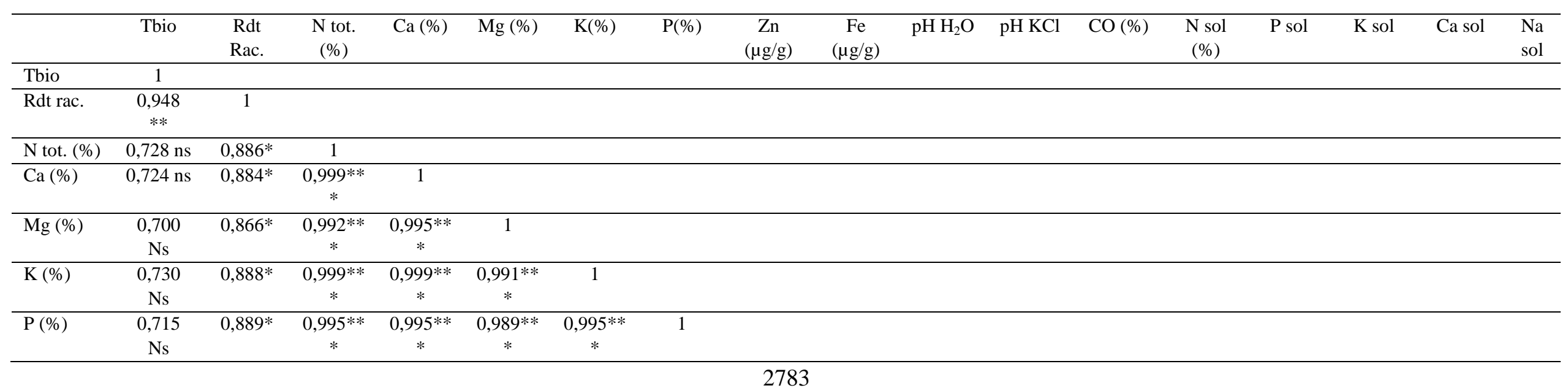


N. C. TEMEGNE et al. / Int. J. Biol. Chem. Sci. 9(6): 2776-2788, 2015

\begin{tabular}{|c|c|c|c|c|c|c|c|c|c|c|c|c|c|c|c|c|}
\hline $\mathrm{Zn}(\mu \mathrm{g} / \mathrm{g})$ & $\begin{array}{c}-0,727 \\
n s\end{array}$ & $\begin{array}{c}-0,885 \\
*\end{array}$ & $\begin{array}{c}-0,999 \\
* * *\end{array}$ & $\begin{array}{c}-0,999 \\
* * *\end{array}$ & $\begin{array}{l}-0,993 \\
* * *\end{array}$ & $\begin{array}{l}-0,999 \\
* * *\end{array}$ & $\begin{array}{l}-0,995 \\
* * *\end{array}$ & 1 & & & & & & & & \\
\hline $\mathrm{Fe}(\mu \mathrm{g} / \mathrm{g})$ & $0,727 \mathrm{~ns}$ & $0,886^{*}$ & $\begin{array}{c}0,999 * * \\
*\end{array}$ & $\begin{array}{c}0,999 * * \\
*\end{array}$ & $\begin{array}{c}0,993 * * \\
*\end{array}$ & $\begin{array}{c}0,999 * * \\
*\end{array}$ & $\begin{array}{c}0,996^{* *} \\
*\end{array}$ & $\begin{array}{c}-0,999 \\
* * *\end{array}$ & 1 & & & & & & & \\
\hline $\mathrm{pH} \mathrm{H}_{2} \mathrm{O}$ & $\begin{array}{c}0,727 \\
\text { Ns }\end{array}$ & $\begin{array}{c}0,896 \\
*\end{array}$ & $\begin{array}{c}0,990 \\
* * *\end{array}$ & $\begin{array}{c}0,988 \\
* * *\end{array}$ & $\begin{array}{c}0,971 \\
* *\end{array}$ & $\begin{array}{c}0,990 \\
* *\end{array}$ & $\begin{array}{c}0,995 \\
* * *\end{array}$ & $\begin{array}{c}-0,989 \\
* * *\end{array}$ & $\begin{array}{c}0,989^{* *} \\
*\end{array}$ & 1 & & & & & & \\
\hline $\mathrm{pH} \mathrm{KCl}$ & $\begin{array}{c}0,728 \\
\mathrm{Ns}\end{array}$ & $\begin{array}{c}0,896 \\
*\end{array}$ & $\begin{array}{c}0,993 \\
* * *\end{array}$ & $\begin{array}{c}0,991 \\
* * *\end{array}$ & $\begin{array}{c}0,976 \\
* *\end{array}$ & $\begin{array}{c}0,993 \\
* * *\end{array}$ & $\begin{array}{c}0,997 \\
* * *\end{array}$ & $\begin{array}{c}-0,992 \\
* * *\end{array}$ & $\begin{array}{c}0,992 \\
* * *\end{array}$ & $\begin{array}{c}0,999 \\
* * *\end{array}$ & 1 & & & & & \\
\hline $\mathrm{CO}(\%)$ & $\begin{array}{c}0,729 \\
\mathrm{Ns} \\
\end{array}$ & $\begin{array}{c}0,887 \\
* \\
\end{array}$ & $\begin{array}{c}0,999 \\
* * * \\
\end{array}$ & $\begin{array}{c}0,999 \\
* * *\end{array}$ & $\begin{array}{c}0,992 \\
* * \\
\end{array}$ & $\begin{array}{c}0,999 \\
* * * \\
\end{array}$ & $\begin{array}{c}0,995 \\
* * *\end{array}$ & $\begin{array}{c}-0,999 \\
* * * \\
\end{array}$ & $\begin{array}{c}0,999 \\
* * * \\
\end{array}$ & $\begin{array}{c}0,990 \\
* * *\end{array}$ & $\begin{array}{c}0,993 \\
* * * \\
\end{array}$ & 1 & & & & \\
\hline N sol (\%) & $\begin{array}{c}0,724 \\
\mathrm{Ns}\end{array}$ & $\begin{array}{c}0,884 \\
*\end{array}$ & $\begin{array}{c}0,999 \\
* * *\end{array}$ & $\begin{array}{c}0,999 \\
* * *\end{array}$ & $\begin{array}{c}0,995 \\
* * *\end{array}$ & $\begin{array}{c}0,999 \\
* * *\end{array}$ & $\begin{array}{c}0,995 \\
* * *\end{array}$ & $\begin{array}{c}-0,999 \\
* * *\end{array}$ & $\begin{array}{c}0,999 \\
* * *\end{array}$ & $\begin{array}{c}0,987 \\
* * *\end{array}$ & $\begin{array}{c}0,991 \\
* * *\end{array}$ & $\begin{array}{c}0,999 \\
* * *\end{array}$ & 1 & & & \\
\hline P sol & $\begin{array}{c}0,724 \\
\mathrm{Ns} \\
\end{array}$ & $\begin{array}{c}0,884 \\
* \\
\end{array}$ & $\begin{array}{c}0,999 \\
* * * \\
\end{array}$ & $\begin{array}{c}0,999 \\
* * *\end{array}$ & $\begin{array}{c}0,995 \\
* * *\end{array}$ & $\begin{array}{c}0,999 \\
* * * \\
\end{array}$ & $\begin{array}{c}0,995 \\
* * *\end{array}$ & $\begin{array}{c}-0,999 \\
* * * \\
\end{array}$ & $\begin{array}{c}0,999 \\
* * * \\
\end{array}$ & $\begin{array}{c}0,987 \\
* * * \\
\end{array}$ & $\begin{array}{c}0,991 \\
* * *\end{array}$ & $\begin{array}{c}0,999 \\
* * * \\
\end{array}$ & $\begin{array}{c}0,999 \\
* * * \\
\end{array}$ & 1 & & \\
\hline K sol & $\begin{array}{c}0,731 \\
\mathrm{Ns} \\
\end{array}$ & $\begin{array}{c}0,888 \\
* \\
\end{array}$ & $\begin{array}{c}0,999 \\
* * *\end{array}$ & $\begin{array}{c}0,999 \\
* * *\end{array}$ & $\begin{array}{c}0,990 \\
* * *\end{array}$ & $\begin{array}{c}0,999 \\
* * *\end{array}$ & $\begin{array}{c}0,995 \\
* * *\end{array}$ & $\begin{array}{c}-0,999 \\
* * * \\
\end{array}$ & $\begin{array}{c}0,999 \\
* * *\end{array}$ & $\begin{array}{c}0,991 \\
* * *\end{array}$ & $\begin{array}{c}0,994 \\
* * *\end{array}$ & $\begin{array}{c}0,999 \\
* * *\end{array}$ & $\begin{array}{c}0,999 \\
* * *\end{array}$ & $\begin{array}{c}0,999 \\
* * * \\
\end{array}$ & 1 & \\
\hline Ca sol & $\begin{array}{c}0,732 \\
\mathrm{Ns} \\
\end{array}$ & $\begin{array}{c}0,885 \\
* \\
\end{array}$ & $\begin{array}{c}0,999 \\
* * * \\
\end{array}$ & $\begin{array}{c}0,999 \\
* * *\end{array}$ & $\begin{array}{c}0,991 \\
* * * \\
\end{array}$ & $\begin{array}{c}0,999 \\
* * *\end{array}$ & $\begin{array}{c}0,993 \\
* * * \\
\end{array}$ & $\begin{array}{c}-0,999 \\
* * * \\
\end{array}$ & $\begin{array}{c}0,999 \\
* * * \\
\end{array}$ & $\begin{array}{c}0,988 \\
* * *\end{array}$ & $\begin{array}{c}0,991 \\
* * *\end{array}$ & $\begin{array}{c}0,999 \\
* * * \\
\end{array}$ & $\begin{array}{c}0,999 \\
* * *\end{array}$ & $\begin{array}{c}0,999 \\
* * * \\
\end{array}$ & $\begin{array}{c}0,999 \\
* * * \\
\end{array}$ & 1 \\
\hline Na sol & $\begin{array}{c}0,750 \\
\mathrm{Ns}\end{array}$ & $\begin{array}{c}0,877 \\
*\end{array}$ & $\begin{array}{c}0,953 \\
* *\end{array}$ & $\begin{array}{c}0,947 \\
* *\end{array}$ & $\begin{array}{c}0,908 \\
*\end{array}$ & $\begin{array}{c}0,956 \\
* *\end{array}$ & $\begin{array}{c}0,946 \\
* *\end{array}$ & $\begin{array}{c}-0,950 \\
* *\end{array}$ & $\begin{array}{c}0,951 \\
* *\end{array}$ & $\begin{array}{c}0,969 \\
* *\end{array}$ & $\begin{array}{c}0,968 \\
* *\end{array}$ & $\begin{array}{c}0,954 \\
* *\end{array}$ & $\begin{array}{c}0,946 \\
* *\end{array}$ & $\begin{array}{c}0,946 \\
* *\end{array}$ & $\begin{array}{c}0,957 \\
* *\end{array}$ & $\begin{array}{c}0,956 \\
* *\end{array}$ \\
\hline
\end{tabular}




\section{DISCUSSION}

Dans les deux sites (Ekona et Ongot), la variété améliorée 8034 est significativement plus riche en éléments minéraux et produit significativement plus de biomasse fraîche totale que les variétés locales (Local Red et Bassa Noire). Elle donne également un rendement en tubercule significativement plus élevé que celles des variétés locales. Ce résultat souligne l'efficacité des travaux d'amélioration du manioc réalisés par l'IRAD du Cameroun dont un des fruits est la variété 8034. En effet, la variété de manioc $\mathrm{N}^{\circ} 8034$ a un potentiel de rendement se situant entre 30 et $40 \mathrm{t} / \mathrm{ha}$ contre 10 à $12 \mathrm{t} / \mathrm{ha}$ seulement pour les variétés traditionnelles cultivées dans les conditions locales du Cameroun (IRAD, 2008). La différence entre ces variétés de manioc pourrait également s'expliquer par l'existence d'une différence génotypique. L'amélioration aurait apporté à la variété 8034, des gènes qui la permettent de se développer convenablement sur les sols pauvres. Abbey et al. (2002) reportent en effet, que la performance des légumes peut être liée à la génétique et à l'influence des facteurs de l'environnement parmi lesquelles la source nutritive, les conditions climatiques, l'état de fertilité du sol, etc. En outre, Diallo et al. (2013) soulignent également que la valeur nutritionnelle d'un légume varie en fonction du cultivar.

La biomasse fraîche totale, le rendement en tubercule, la composition des feuilles en azote total, calcium, magnésium, potassium, phosphore et en fer sont significativement plus importants à Ekona qu'à Ongot. Dans les deux sites d'étude, les valeurs des quantités d'éléments nutritifs contenues dans les feuilles de manioc démontrent que le manioc est une plante épuisante du sol. La composition minérale du sol des sites pourrait expliquer ces résultats. Le $\mathrm{pH}$ du sol d'Ekona $(5,45)$ est moins acide que celui d'Ongot $(4,70)$. L'acidité élevée du sol influence donc négativement le rendement du manioc. En effet, Ongot possède un sol ferralitique (ferralsols) tandis que le sol
d'Ekona est volcanique. Les sols volcaniques sont connus pour être plus riches en éléments minéraux et moins acides comparés aux sols ferralitiques (Nanzyo, 2002). Le manioc supporte des sols de $\mathrm{pH}$ compris entre 3,5 et 7,8 (Fageria et al., 2010); le pH du sol des deux sites est donc propice à la culture du manioc. Les résultats de l'analyse du sol d'Ongot sont similaires à ceux de Yerima et van Ransk (2005) qui ont montré que les sols ferralitiques du Cameroun étaient généralement acides, avec des faibles teneurs en carbone organique, azote total, et une déficience en potassium échangeable et en phosphore disponible. L'analyse du sol (Tableau 1) montre d'ailleurs que la matière organique du sol est plus élevée à Ekona qu'à Ongot. Bien que le manioc soit connu pour croître dans les sols très pauvres (Hauser et al., 2006 ; Echebiri et Edaba, 2008), un sol riche en éléments minéraux favorise sa croissance.

Les teneurs en phosphore disponible et en potassium plus élevées dans le sol d'Ekona que dans celui d'Ongot pourraient également expliquer les rendements plus importants à Ekona. En effet, le phosphore est essentiel pour maximiser et soutenir durablement le rendement potentiel des cultures dans les systèmes de cultures en continu (Kuang et al., 2005). Il joue un rôle prépondérant dans le développement des racines tubéreuses du manioc (Ukaoma et Ogbonnaya, 2013). Le potassium est également essentiel pour la culture du manioc. Il joue un rôle déterminant dans le régime hydrique des plantes et permet une meilleure absorption des éléments nutritifs par le manioc (Adjanohoun, 2006).

L'analyse du sol a montré que le sol d'Ekona est plus riche en phosphore, azote total, potassium, calcium et sodium en comparaison à celui d'Ongot. Ce résultat explique la corrélation positive et très hautement significative entre la composition minérale du sol des deux sites et celle des feuilles de manioc. La pluviométrie plus importante à Ekona (3 076 mm) qu'à Ongot (1 $617 \mathrm{~mm}$ ) pourrait également expliquer le 
rendement en biomasse fraîche totale plus élevé à Ekona. En effet, les précipitations abondantes et régulières favorisent la croissance végétative des cultures et boostent par conséquent leur rendement. Néanmoins, une moyenne de précipitations comprise entre 1000 et $2000 \mathrm{~mm}$ par an est généralement satisfaisante (Sheets et al., 2012; Howeler et al., 2013) pour la culture du manioc.

D'après le tableau des concentrations standards en éléments nutritifs des feuilles de manioc proposé par Howeler et Phien (2000), la teneur en calcium des feuilles de manioc est toxique, les teneurs en fer et en magnésium sont élevées, la teneur en zinc est suffisante, les teneurs en phosphore et potassium sont déficientes et la teneur en azote est très déficiente dans les deux sites d'étude. En effet, les travaux d'Adjanohoun (2006) ont montré que la quantité d'azote extraite par les plantes de manioc pour l'obtention des rendements les plus élevés, (au moins 20 t.ha ${ }^{1}$ ), est supérieure à $100 \mathrm{~kg}^{-h^{-1}}$, celle du phosphore supérieure à $60 \mathrm{~kg} \cdot \mathrm{ha}^{-1}$ et enfin celle de du potassium supérieure à $190 \mathrm{~kg}$.ha1. Selon ces mêmes auteurs, en comparaison au tableau de classification des caractéristiques du sol en fonction des besoins nutritifs du manioc (Howeler et Phien, 2000), le $\mathrm{pH}$ et le phosphore du sol des deux sites est moyen. La teneur en matière organique du sol est moyenne à Ongot mais élevée à Ekona. À Ongot, la teneur en calcium du sol est faible tandis qu'elle est moyenne à Ekona.

\section{Conclusion}

L'objectif de ce travail était de déterminer l'influence de la composition chimique du sol sur le rendement et la composition nutritive des feuilles de manioc dans les Régions du Centre (Ongot) et du SudOuest (Ekona) du Cameroun. Les résultats obtenus au terme de cette étude révèlent que comparé aux variétés traditionnelles (Bassa Noire et Local Red), la variété 8034 possède des qualités agronomiques qui lui permettent de produire un rendement significativement plus élevé et des légumes (feuilles) significativement plus riches en éléments nutritifs (azote, calcium, phosphore, magnésium, potassium, zinc et fer) dans les conditions locales. Le sol de la localité d'Ekona est plus fertile que celui de la zone d'Ongot. La richesse des feuilles et des tubercules du manioc en éléments nutritifs est proportionnelle à celle du sol en éléments minéraux.

\section{CONFLITS D'INTÉRÊT}

Les auteurs déclarent qu'ils ont aucun conflit d'intérêts.

\section{CONTRIBUTIONS DES AUTEURS}

L'auteur CTN a recueilli les données et rédigé le projet du manuscrit. L'auteur FNA a élaboré le protocole de collecte des données, facilité le travail sur le terrain et corrigé le projet du manuscrit. L'auteur AFK a lu le projet du manuscrit et a fait d'importantes contributions.

\section{REMERCIEMENTS}

Les auteurs apprécient le soutien logistique de l'IRAD d'Ekona et de Nkolbisson.

\section{REFERENCES}

Abbey L, Joyce DC, Akad J, Smith B. 2002. Genotype, sulphur and nutrition and soil types effects on growth and dry matter production of spring onion. $J$. Hortic. Sci. Biotech., 77: 340-345.

Adjanohoun A. 2006. Nutrition du manioc sous différentes combinaisons de NPK au Sud du Bénin. B.R.A.B. (Bulletin de la Recherche Agronomique du Bénin), 52: 1-6.

Atangana A. 1998. Rapport d'étude sur les activités agricoles dans le village d'Ongot, Province du Centre, Cameroun. IRAD: Yaoundé.

Betiku E, Alade OS. 2011. Investigation of effects of different cassava cultivars with respect to hydrogen cyanide content on their starch hydrolysis. Int. J. Biol. Chem. Sci., 5(6): 2362-2370. 
DOI: http://dx.doi.org/10.4314/ijbcs. v5i6.16

Diallo Y, Gueye MT, Sakho M, Darboux PG, Kane A, Barthelemy J-P, Lognay G. 2013. Importance nutritionnelle du manioc et perspectives pour l'alimentation de base au Sénégal. Biotechnol. Agron. Soc. Environ., 17(4): 634-643. URL : http://popups.ulg.ac.be/17804507/index.php?id=10440.

Dury S. 2001. Consumption of cassava derived products in southern cities in Cameroon. CIRAD/IITA. Document de travail.

Echebiri RN, Edaba ME. 2008. Production and utilization of cassava Nigeria: Prospects for food security and infant nutrition. J. Prod. Agric. Technol., 4: 35-52. ISSN: 0794-5213.

Fageria NK, Baligar VC, Jones CA. 2010. Growth and Mineral Nutrition of Field Crops, $3^{\text {rd }}$ edn. CRC Press.

Famurewa JAV, Oluwamukomi MO, Alaba JO. 2013. Effect of different drying methods on the physicochemical characteristics of cassava flour ("pupuru"). Int. J. Biol. Chem. Sci., 7(2): 832-839. DOI : http://dx.doi.org/10.4314/ijbcs.v7i2.38

FAO (Food and Agriculture Organization). 2000. Défendre la cause du manioc. FAO. http://www.fao.org/nouvelle/ 2000/000405-f.htm.

FAO (Food and Agriculture Organization). 2003. Gestion de la fertilité des sols pour la sécurité alimentaire en Afrique subsaharienne. FAO: Rome.

FAO (Food and Agriculture Organization). 2015. FAOSTAT. http://faostat. fao.org/, consulté le 25/10/2014.

Gnonlonfin GJB, Koudande DO, Sanni A, Brimer L. 2011. Farmers' perceptions on characteristics of cassava (Manihot esculenta Crantz) varieties used for chips production in rural areas in Benin, West Africa. Int. J. Biol. Chem. Sci., 5(3): 870-879.
Hauser S, Nolte C, Carsky RJ. 2006. What role can planted fallows play in the humid and sub-humid zones of West and Central Africa? Nutr. Cycl. Agroecosyst., 76: 297-318. DOI: 10.1007/s10705-005-5630-4.

Howeler RH, Phien T. 2000. Integrated nutrient management for more sustainable cassava production in Vietnam. In Proceedings of the Vietnamese Cassava Workshop on Progress in Cassava Research and Extension in Vietnam: Ho Chi Minh City; 12-54.

Howeler RH, Lutaladio N, Thomas G. 2013. Save and Grow: Cassava - A Guide to Sustainable Production Intensification. FAO: Rome.

IRAD. 2008. Rapport national sur l'état des ressources phytogénétiques pour l'alimentation et l'agriculture. IRAD: Yaoundé.

IRAD. 2013. Diffusion des Nouvelles Technologies Agricoles en Afrique DONATA (Composante II de PSTAD). IRAD (Institut de la Recherche Agronomique pour le Développement) Cameroun, Rapport annuel d'activités 2013 : Yaoundé.

Koko CA, Kouame BK, Assidjo E, Amani G. 2014. Characterization and utilization of fermented cassava flour in breadmaking and placali preparation. Int. J. Biol. Chem. Sci. 8(6): 24782493. DOI : http://dx.doi.org/ 10.4314/ijbcs.v8i6.11

Kuang RB, Liao H, Yan XL, Dong YS. 2005. Phosphorus and nitrogen interactions in field-grown soybean as related to genetic attributes of root morphological and nodular traits. J. Int. Plant Biol., 47: 549-559. DOI: $10.1111 /$ j.17447909.2005.00072.x

Lebot V. 2009. Tropical Roots and Tuber Crops: Cassava, Sweet Potato, Yams and Aroids. CAB International: Oxford. 
Nanzyo M. 2002. Unique properties of volcanic ash soils. Global Environmental Research, 6(2): 83-97.

Ngome AF, Amougou MFC, Tata PI, Ndindeng SA, Mfopou MYC, Mapiemfu-Lamare D, Njeudeng TS. 2013. Effects of cassava cultivation on soil quality indicators in the humid forest of Cameroon. Greener J. Agric. Sci., 3: 451-457. www.gjournals.org.

Okalebo JR, Gathua KW, Woomer PL. 2002. Laboratory Methods of Soil and Plant Analysis: A Working Manual. TSBF : Nairobi.

PNDRT (Programme National de Développement des Racines et Tubercules). 2003. Analyse de la Demande et des Marchés et Propositions pour Améliorer l'Efficacité de la Commercialisation des Racines et Tubercules et Augmenter le Revenu des Producteurs. MINADER : Yaoundé.

Sheets P, Lentz D, Piperno D, Jones J, Dixon C, Maloof G, Hood A. 2012. Ancient manioc agriculture south of the Cerén village, El Salvador. Lat. Am. Antiq., 23(3): 259-281.
Tricoche B, David-Benz H, Song J-E. 2008. L'organisation de la filière manioc au Cameroun: des modes de coordination pour régir les incertitudes de marché. Atelier REPARAC, Juin 2008, Yaoundé, Cameroun; pp. 13. <hal00415315>.

Tsalefac M, Ngoufo R, Nkwambi W, Djoumessi Tatsangue E, Lengue Fobissie B. 2003. Fréquences et quantités des précipitations journalières sur le territoire camerounais. Publication de l'Association Internationale de Climatologie, $\mathbf{1 5}$ : 359-367.

Ukaoma AA, Ogbonnaya CI. 2013. Effect of inorganic mineral nutrition on tuber yield of cassava (Manihot esculenta Crantz) on marginal ultisol of South Eastern Nigeria. Academia Journal of Agricultural Research, 1(9): 172-179. DOI:

http://dx.doi.org/10.15413/ajar.2013.01 34.

Yerima BP, van Ransk K. 2005. Major Soil Classification Systems Used in the Tropics: Soils of Cameroon. Trafford Publishing: USA. 